\title{
Avaliação dos resultados do tratamento cirúrgico das fraturas-luxações da extremidade proximal do antebraço no adulto*
}

\author{
Evaluation of the outcome in fracture-dislocation \\ surgeries in the proximal end of the adult forearm
}

\author{
Sérgio luiz Checchia ${ }^{1}$, Alberto Naoki Miyazaki², Marcelo Fregoneze ${ }^{3}$, Pedro Doneux Santos 4 , \\ luciana Andrade da Silva ${ }^{4}$, Eduardo Yoshiaki NaKandakari ${ }^{5}$, Guilherme do Val Sella ${ }^{5}$, MÁrcio Schiefer ${ }^{5}$
}

\section{RESUMO}

Objetivo: Avaliar os resultados obtidos no tratamento das fraturas-luxações da extremidade proximal do antebraço (FLEPA), procurando identificar fatores que possam influenciar no prognóstico desse tipo de lesão traumática. Métodos: De outubro de 1994 a dezembro de 2005, 30 pacientes com FLEPA foram submetidos ao tratamento cirúrgico. Em todos os casos, o seguimento ambulatorial mínimo foi de 12 meses. As lesões foram classificadas pelo

* Trabalho realizado no Departamento de Ortopedia e Traumatologia da Faculdade de Ciências Médicas da Santa Casa de São Paulo (DOT-FCMSCSP), Pavilhão "Fernandinho Simonsen" (Diretor: Prof. Dr. Cláudio Santili), São Paulo (SP) - Brasil.

1. Professor Adjunto e Chefe do Grupo de Ombro e Cotovelo do Departamento de Ortopedia e Traumatologia da Faculdade de Ciências Médicas da Santa Casa de São Paulo, São Paulo (SP), Brasil.

2. Professor Assistente e Assistente do Grupo de Ombro e Cotovelo do Departamento de Ortopedia e Traumatologia da Faculdade de Ciências Médicas da Santa Casa de São Paulo, São Paulo (SP), Brasil.

3. Professor Assistente e Assistente do Grupo de Ombro e Cotovelo do Departamento de Ortopedia e Traumatologia da Faculdade de Ciências Médicas da Santa Casa de São Paulo, São Paulo (SP), Brasil.

4. Assistente do Grupo de Ombro e Cotovelo do Departamento de Ortopedia e Traumatologia da Faculdade de Ciências Médicas da Santa Casa de São Paulo, São Paulo (SP), Brasil.

5. Estagiário do Grupo de Ombro e Cotovelo do Departamento de Ortopedia e Traumatologia da Faculdade de Ciências Médicas da Santa Casa de São Paulo, São Paulo (SP), Brasil.

Endereço para correspondência: Santa Casa de Misericórdia de São Paulo, Departamento de Ortopedia e Traumatologia, "Pavilhão Fernandinho Simonsen”, Rua Dr. Cesário Mota Jr., 112 - 01221-020 - São Paulo (SP), Brasil. Tel./ fax: (11) 3222-6866. E-mail: ombro@ ombro.med.br; site: www.ombro.med.br Recebido em 13/3/07. Aprovado para publicação em 24/7/07.

Copyright RBO2007 método de Chick et al. A média de idade foi de 48 anos, com mínima de 25 e máxima de 76 anos. Os mecanismos de trauma foram: queda da própria altura em 17 pacientes $(57 \%)$, acidentes de via pública em 11 casos (37\%), trauma direto em um caso (3\%) e agressão física em um caso $(3 \%)$. As fraturas expostas corresponderam a oito casos $(27 \%)$. Realizou-se a análise estatística pelo teste exato de Fisher para avaliar os seguintes dados: exposição do foco de fratura e resultados; sexo e resultados; mecanismo de trauma e resultados; idade e mecanismo de trauma; sexo e mecanismo de trauma. Resultados: Em 11 casos (37\%) os resultados foram satisfatórios e em 19 casos (63\%), insatisfatórios. Estabeleceu-se apenas relação estatística significativa entre exposição da fratura e resultados. Conclusão: Os tratamentos das FLEPA têm, em sua maioria, resultados insatisfatórios, principalmente nas fraturas expostas, fato estatisticamente significativo neste estudo. Não foi possível definir outros fatores prognósticos. Encontrou-se tendência a melhores resultados nos pacientes do sexo feminino, nos idosos e nas vítimas de traumas de baixa energia.

Descritores - Traumatismos do antebraço/cirurgia; Resultado de tratamento; Estudos retrospectivos; Adulto

\section{ABSTRACT}

Objective: To evaluate results obtained in the treatment of fracture-dislocation of the proximal end of the forearm (FLEPA), in an attempt at identifying factors that may influence the prognosis of this type of traumatic lesion. Methods: From October 1994 to December 2005, 30 patients with FLEPA were submitted to surgical treatment. In all cases, 
minimum office follow-up lasted 12 months. The lesions were classified according to the method of Chick et al. Mean age was 48 years, with minimum age of 25 , and maximum age of 76 years. Traumas were caused by: falling to the ground in 17 patients (57\%), street accidents in 11 cases (37\%), direct trauma in one case (3\%), and bodily aggression in one case (3\%). Open fractures were seen in eight cases (27\%). Statistical analysis was run by the exact Fisher test to evaluate the following data: exposure of the fracture focus and results; gender and results; mechanism of the trauma and results; age and mechanism of trauma; gender and trauma mechanism. Results: In 11 cases (37\%), results were satisfactory, and in 19 cases (63\%) results were unsatisfactory. They only established the significant statistical ratio between fracture exposure and results. Conclusion: Most FLEPA treatments have unsatisfactory results, particularly in cases of open fractures. Other prognosis factures could not be defined. The authors identified a trend towards better results in female patients, among the elderly, and in the victims of low energy traumas.

Keywords - Forearm injuries/surgery; Treatment outcome;
Retrospective studies; Adult

\section{INTRODUÇÃO}

Segundo Chick et al, as lesões traumáticas complexas da extremidade proximal dos ossos do antebraço são de dois tipos principais: a fratura-luxação de Monteggia (FLM) e a luxação transolecraniana ou fratura-luxação da extremidade proximal dos ossos do antebraço (FLEPA) $)^{(1)}$. As FLEPA são afecções relativamente incomuns ${ }^{(2)}$. O tratamento definido é cirúrgico, com fixação interna estável e reabilitação precoce, com o objetivo de evitar a rigidez $\operatorname{articular}^{(3)}$. Para tanto, é necessário diagnóstico preciso, já que erros em diagnosticar essas lesões ocorrem em até $50 \%$ dos $\operatorname{casos}^{(4)}$.

A ocorrência de fraturas cominutas devido à íntima proximidade dos músculos com a cápsula articular aumenta o risco de rigidez pós-traumática do cotovelo, complicação que pode ser encontrada em $20 \%$ das luxações do cotovelo, $38 \%$ das fraturas-luxações e $10 \%$ das fraturas da cabeça do rádio, mesmo nos casos em que a reconstrução anatômica foi obtida e o programa de recuperação funcional foi adotado corretamen$\mathrm{te}^{(5)}$.

O objetivo deste trabalho é avaliar os resultados obtidos no tratamento das FLEPA, procurando encontrar fatores prognósticos.

\section{MÉTODOS}

De outubro de 1994 a dezembro de 2005, 30 pacientes, sendo 14 do sexo masculino (47\%) e 16 do feminino (53\%) com FLEPA, foram operados no Grupo de Cirurgia de Ombro e Cotovelo do Departamento de Ortopedia e Traumatologia da Faculdade de Ciências Médicas da Santa Casa de São Paulo - "Pavilhão Fernandinho Simonsen". Todos os casos possuíam radiografias pré e pós-operatórias e seguimento ambulatorial mínimo de 12 e máximo de 84 meses, com média de 33 meses.

Os pacientes foram relacionados de acordo com sexo, idade, lado acometido, lado dominante, profissão e presença ou não de anormalidades neurológicas e/ou vasculares pré e pósoperatórias. As lesões foram classificadas pelo método de Chick et $a l^{(1)}$, que leva em consideração a localização da fratura na ulna, a direção do desvio da cabeça do rádio, a presença ou não de fratura da cabeça do rádio e fratura do processo coronóide. As fraturas da ulna podem ser classificadas como epifisária (tipo I), epifisio-metafisária (tipo II) e metafisária ou diafisária (tipo III). A direção do desvio do rádio podem ser anterior (A) ou posterior (P) (figura 1). Em relação ao processo coronóide, verificamos: ausência de fratura $(\mathrm{C} 0)$, fratura no ápice $(\mathrm{C} 1)$, fratura de menos de $50 \%(\mathrm{C} 2)$, fratura de mais de $50 \%$ (C3) e fratura cominutiva (C4) (figura 2). Quanto à extremidade proximal do rádio, observamos: ausência de fratura (R0), fratura sem desvio (R1), fratura marginal com desvio (R2), fratura cominutiva ou do colo (R3) (figura 3).

A média de idade foi de 49 anos, com mínima de 25 e máxima de 76 anos. Os pacientes do sexo feminino tinham média de idade de 60 anos (de 33 a 76 anos) e os do sexo masculino, de 35 anos (de 25 a 63 anos). O lado dominante foi acometido em 15 pacientes (50\%) (tabela 1).

Os mecanismos de trauma foram queda em 17 pacientes (57\%), acidentes de via pública em 11 casos (37\%), trauma direto em um caso (3\%) e agressão física em um caso (3\%). As fraturas expostas corresponderam a oito pacientes (27\%). Entre os pacientes, 17 sofreram fratura da cabeça do rádio (55\%), 16 fratura do processo coronóide (57\%). Em 11 pacientes ocorrera luxação da cabeça do rádio (37\%) (figura 4) e em 20 a lesão era posterior (63\%) (figura 5) (tabela 1). Um paciente sofreu variante complexa da lesão de Monteggia posterior (Bado II), caracterizada por luxação ulnoumeral associada a fratura metaepifisária da ulna, fratura cominutiva da cabeça do rádio e fratura da base do processo coronóide (figura 6).

Não foram observadas complicações imediatas decorrentes do tratamento realizado. 


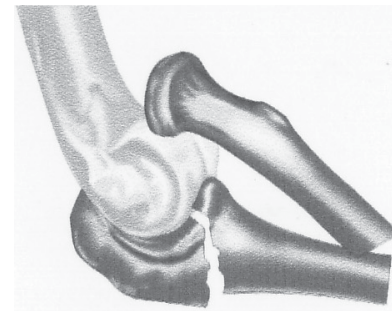

A

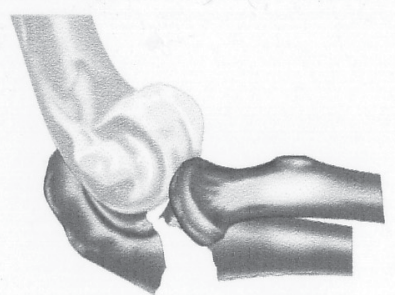

D

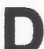

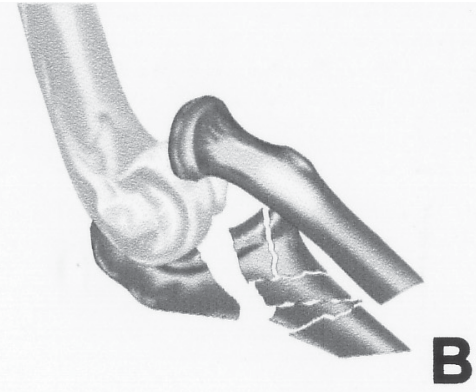

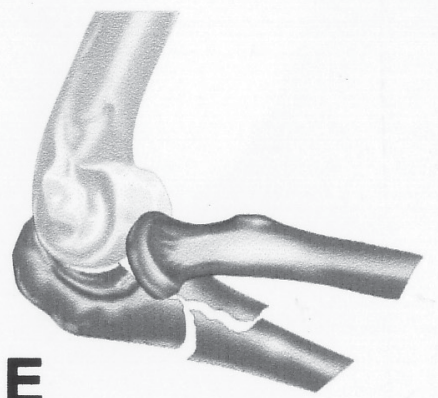

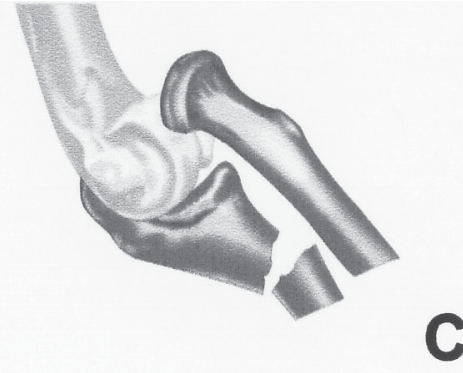

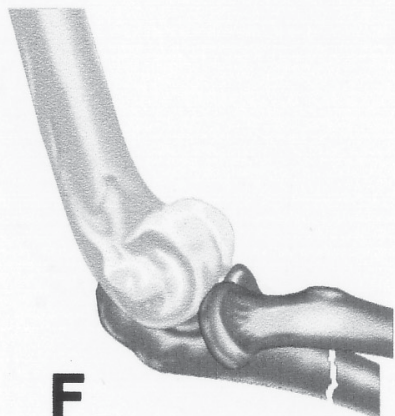

Figura 1 - (A, B e C) Fratura do tipo I (A), tipo II (B) e do tipo III (C), com desvio anterior do rádio. (D, E e F) Fratura do tipo I (D), tipo II (E) e tipo III (F), com desvio posterior do rádio.

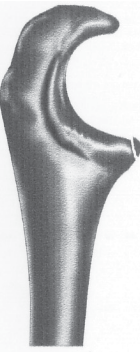

A

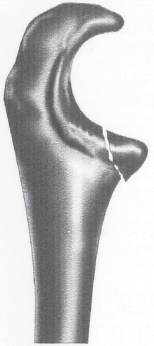

B

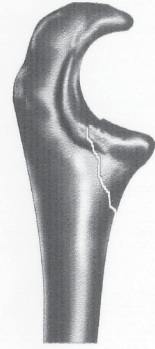

C

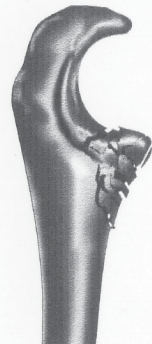

D

Figura 2 - Fratura do processo coronóide: (A) ápice (C1), (B) menos de $50 \%$ (C2), (C) mais de $50 \%$ (C3) e (D) multifragmentar (C4).
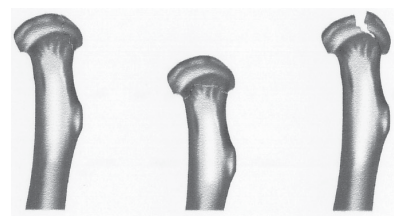

A

B

C

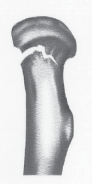

D

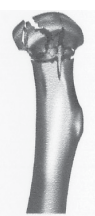

E

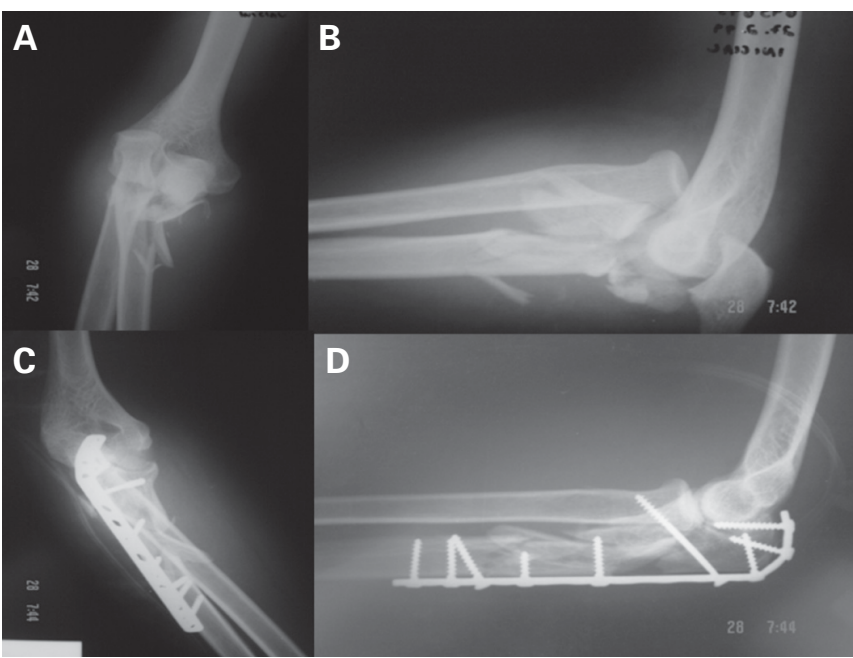

Figura 4 - Caso 21. (A) Imagem radiográfica de frente evidenciando fratura na região proximal do antebraço direito - tipo IIAC4R0 (Chick). (B) Imagem radiográfica em perfil evidenciando luxação anterior da cabeça do rádio. Imagens de radiografia no pós-operatório imediato. (C) Frente e (D) Perfil.

Figura 3 - Fratura da extremidade proximal do rádio sem desvio (A e B), marginal com desvio (C e D) e multifragmentar ou do colo (E e F). 
TABELA 1

Dados epidemiológicos dos pacientes

\begin{tabular}{|c|c|c|c|c|c|c|c|}
\hline Número & Nome & $\begin{array}{l}\text { Idade } \\
\text { (anos) }\end{array}$ & Sexo & Dominância & $\begin{array}{c}\text { Mec. } \\
\text { trauma }\end{array}$ & Exposta & Classificação \\
\hline 1 & F.P.L. & 67 & $f$ & + & atrop & - & II/A/C4/R3 \\
\hline 2 & M.E. & 33 & $\mathrm{~m}$ & - & ac moto & + & II/A/C3/R0 \\
\hline 3 & A.G.O. & 36 & $\mathrm{~m}$ & - & queda & + & II/P/C3/R3 \\
\hline 4 & J.S.S. & 32 & $\mathrm{~m}$ & - & ac auto & - & III/A/CO/RO \\
\hline 5 & O.R.P. & 25 & $\mathrm{~m}$ & - & ac auto & - & II/A/C4/R3 \\
\hline 6 & M.S.C. & 36 & $f$ & - & ac auto & + & II/P/C1/R0 \\
\hline 7 & A.M.C.M. & 58 & $f$ & - & queda & - & $\mathrm{III} / \mathrm{P} / \mathrm{CO} / \mathrm{R} 2$ \\
\hline 8 & F.R.M. & 30 & $\mathrm{~m}$ & - & ac auto & + & $\mathrm{III} / \mathrm{P} / \mathrm{C} 3 / \mathrm{RO}$ \\
\hline 9 & G.P.S. & 30 & $\mathrm{~m}$ & - & atrop & + & II/A/C3/R0 \\
\hline 10 & C.C.G. & 73 & $f$ & - & queda & - & III/P/CO/RO \\
\hline 11 & A.E.P. & 67 & $f$ & + & queda & - & III/P/C0/R3 \\
\hline 12 & S.D. & 38 & $f$ & + & ac auto & - & $\mathrm{II} / \mathrm{P} / \mathrm{CO} / \mathrm{RO}$ \\
\hline 13 & M.T.N.S. & 63 & $f$ & - & queda & - & III/P/C2/R3 \\
\hline 14 & M.B.S. & 58 & $f$ & + & queda & - & III/P/C3/R3 \\
\hline 15 & V.J.P. & 36 & $\mathrm{~m}$ & + & queda & - & II/P/C3/R3 \\
\hline 16 & T.C.L.P. & 33 & $f$ & - & queda & - & III/P/C0/R1 \\
\hline 17 & O.A.S. & 63 & $\mathrm{~m}$ & - & ac auto & + & III/A/CO/RO \\
\hline 18 & D.A.N. & 67 & $f$ & - & queda & - & $\mathrm{III} / \mathrm{P} / \mathrm{CO} / \mathrm{R} 2$ \\
\hline 19 & E.B. & 74 & $f$ & + & queda & - & III/P/C2/R1 \\
\hline 20 & R.L.B.S. & 24 & $\mathrm{~m}$ & + & ac moto & + & $\mathrm{II} / \mathrm{A} / \mathrm{CO} / \mathrm{RO}$ \\
\hline 21 & D.M. & 42 & $\mathrm{~m}$ & + & ac auto & - & II/A/C4/R0 \\
\hline 22 & L.S. & 49 & $\mathrm{~m}$ & + & queda & - & $\mathrm{II} / \mathrm{A} / \mathrm{C} 1 / \mathrm{R} 0$ \\
\hline 23 & A.P. & 73 & $f$ & + & queda & - & II/P/C0/R2 \\
\hline 24 & R.M.P.L. & 35 & $\mathrm{~m}$ & - & agressão & + & $\mathrm{III} / \mathrm{P} / \mathrm{CO} / \mathrm{RO}$ \\
\hline 25 & W.N.P.J. & 25 & $\mathrm{~m}$ & + & queda & - & $\mathrm{III} / \mathrm{P} / \mathrm{C} 1 / \mathrm{R} 3$ \\
\hline 26 & A.M.F.C. & 46 & $f$ & - & queda & - & II/P/C3/R3 \\
\hline 27 & R.T.C.G. & 67 & $f$ & + & queda & - & $\mathrm{III} / \mathrm{P} / \mathrm{CO} / \mathrm{RO}$ \\
\hline 28 & R.C.M. & 68 & $f$ & + & queda & - & II/A/C3/R0 \\
\hline 29 & G.L.R.J. & 53 & $\mathrm{~m}$ & + & queda & - & $\mathrm{I} / \mathrm{P} / \mathrm{CO} / \mathrm{R} 2$ \\
\hline 30 & O.T.A. & 76 & $f$ & + & queda & - & III/A/CO/R2 \\
\hline
\end{tabular}

Legendas: m: masculino, f: feminino; mec.: mecanismo; atrop.: atropelamento; ac.: acidente; A: anterior; P: posterior; C: coronóide; R: cabeça do rádio.

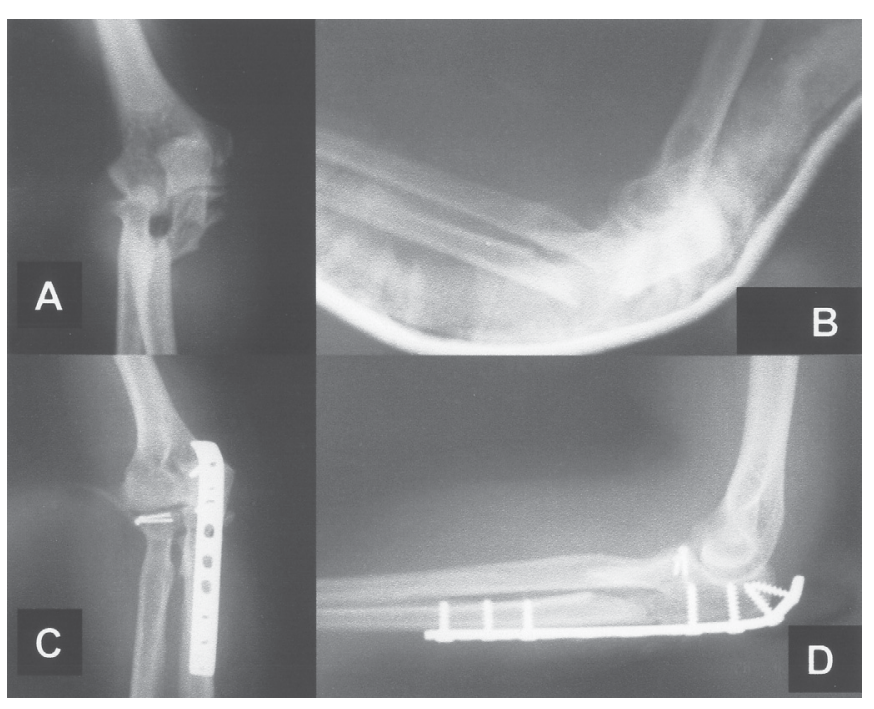

Todos os pacientes foram operados através da via de acesso posterior, sendo a osteossíntese da ulna feita em todos os casos com placa e parafusos de $3,5 \mathrm{~mm}$, à exceção de um caso (3\%), em que a técnica empregada foi a banda de tensão na ulna. Enxerto ósseo foi utilizado em cinco pacientes (17\%) que apresentavam fratura multifragmentar e em um, cuja fratura evoluiu para pseudartrose. Das 16 fraturas da cabeça do rádio, 15 foram operadas pelos seguintes procedimentos: sete ressecções (46\%), seis osteossínteses internas com material de minimicrofragmentos (40\%), uma artroplastia (7\%) e uma

Figura 5 - Caso 14. (A) Imagem radiográfica de frente evidenciando fratura na região proximal do antebraço direito - tipo IIIPCOR3 (Chick). (B) Imagem radiográfica em perfil evidenciando luxação posterior da cabeça do rádio. Imagens de radiografia no pós-operatório imediato. (C) Frente e (D) Perfil. 
ressecção de fragmento fraturado da cabeça do rádio (7\%). Em um caso, por apresentar mínimo desvio da fratura da cabeça do rádio, não houve indicação cirúrgica (tabela 2).
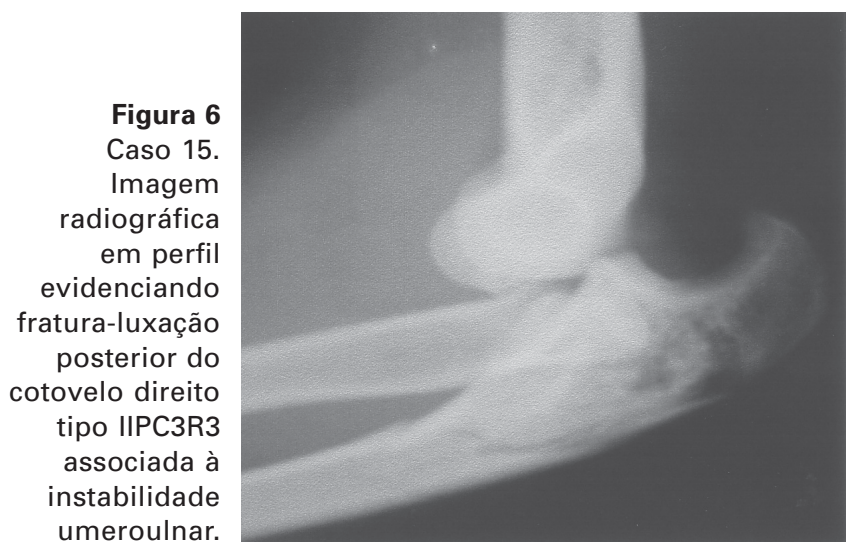

Dos oito pacientes (27\%) com fratura exposta, em três (38\%) foi realizada fixação externa como método de estabilização inicial. Posteriormente, as fraturas foram fixadas com osteossíntese interna definitiva após a melhora das condições de partes moles; o período entre os procedimentos variou de duas a nove semanas, com média de sete semanas. Nos demais casos, devido à pequena lesão de partes moles, a osteossíntese interna foi possível no primeiro tempo cirúrgico (tabela 2).

Com relação aos resultados, agrupamos como satisfatórios os de todos os pacientes considerados excelentes e bons e, como insatisfatórios, aqueles considerados regulares e ruins, conforme os critérios da American Medical Association (AMA) modificado por Bruce et al(6).

Para a realização da análise estatística, dividimos os pacientes em dois grupos quanto à faixa etária, sendo um grupo acima de 55 anos e outro grupo abaixo de 55 anos de idade.

TABELA 2

Relação dos procedimentos cirúrgicos realizados em cada paciente

\begin{tabular}{|c|c|c|c|}
\hline Caso & Primeira cirurgia & Segunda cirurgia & Terceira cirurgia \\
\hline 1 & RAFI + ressecção cab. rádio & - & - \\
\hline 2 & fix. externa & RAFI + enxerto & RMS \\
\hline 3 & RAFI + ressecção cab. rádio & RMS & - \\
\hline 4 & RAFI & RMS + RAFI & - \\
\hline 5 & RAFI & LAP + ressecção cab. rádio & - \\
\hline 6 & RAFI & enxerto & RMS \\
\hline 7 & RAFI + ressecção cab. rádio & encos & - \\
\hline 8 & RAFI & RAFI + enxerto & - \\
\hline 9 & fix. externa & RAFI & - \\
\hline 10 & RAFI & - & - \\
\hline 11 & RAFI + fix cab. rádio & - & - \\
\hline 12 & RAFI & - & - \\
\hline 13 & fix. externo & RAFI + artroplastia cab. rádio & RMS \\
\hline 14 & RAFI + fix. cab. rádio & RMS & - \\
\hline 15 & RAFI + fix. cab. rádio & - & - \\
\hline 16 & RAFI & - & - \\
\hline 17 & fix. externo & RMS + RAFIRAFI + enxerto & \\
\hline 18 & RAFI + fix. cab. rádio & - & - \\
\hline 19 & RAFI + retirada frag. cab. rádio & RMS & - \\
\hline 20 & RAFI & - & - \\
\hline 21 & RAFI & ressecção SRUP & RMS + LAP \\
\hline 22 & RAFI + enxerto & $\mathrm{RMS}+\mathrm{BT}+$ enxerto & - \\
\hline 23 & RAFI + fix. cab. rádio & RMS & - \\
\hline 24 & RAFI & LAP & RMS \\
\hline 25 & RAFI + ressecção cab. rádio & - & - \\
\hline 26 & RAFI + ressecção cab. rádio & - & - \\
\hline 27 & RAFI & - & - \\
\hline 28 & RAFI & - & - \\
\hline 29 & BT ulna + fix. rádio & - & - \\
\hline 30 & RAFI + ressecção cab. rádio & - & - \\
\hline
\end{tabular}

Legendas: RAFI: redução aberta e fixação interna; fix.: fixação; cab.: cabeça; frag.: fragmento; BT: banda de tensão; RMS: retirada de material de síntese; LAP: liberação anterior e posterior; SRUP: sinostose radioulnar proximal. 
Em relação ao mecanismo de trauma, dividimos em alta energia aqueles que sofreram acidente automobilístico, motociclístico ou atropelamento e baixa energia aqueles que sofreram queda ao solo.

Realizamos a análise estatística pelo teste exato de Fisher para avaliar os seguintes dados: exposição do foco de fratura e os resultados; sexo e resultados; mecanismo de trauma e resultados; idade e mecanismo de trauma; sexo e mecanismo de trauma. Foi considerado estatisticamente significativo quando $p<0,05$.

\section{RESULTADOS}

De acordo com os critérios da American Medical Association (AMA) modificado por Bruce et al ${ }^{(6)}$, verificamos que em sete pacientes (23\%) o resultado foi excelente; em quatro, bom
(14\%); em sete, regular (23\%); e em 12, ruim (40\%), com a pontuação variando entre 50 e 100 , totalizando $37 \%$ de casos satisfatórios e $63 \%$ de insatisfatórios (tabela 3).

Os 12 casos considerados como ruins apresentavam diminuição da amplitude de movimento. Entre esses pacientes, três informaram ter limitações para as atividades da vida diária (AVD), queixou-se de dor leve que não comprometia a AVD e um de dor que interferia com as AVD.

Entre os sete casos considerados como regulares, todos possuíam limitação da amplitude de movimento, um paciente relatou limitações da AVD e dois queixaram-se de dor que interferia com AVD.

Apenas um paciente, dos 11 considerados como tendo tido bom e excelente resultado, relatou dor leve que não comprometia as AVD.

TABELA 3

Tempo de seguimento e resultados funcionais obtidos segundo os critérios da American Medical Association (AMA) modificados por Bruce et al

\begin{tabular}{|c|c|c|c|c|c|}
\hline \multirow[t]{2}{*}{ Caso } & \multirow{2}{*}{$\begin{array}{l}\text { Seguimento } \\
\text { (meses) }\end{array}$} & \multirow[t]{2}{*}{ Flexão/Extensão } & \multirow[t]{2}{*}{ Pronação/Supinação } & \multicolumn{2}{|c|}{ Avaliação funcional } \\
\hline & & & & Pontuação & Avaliação \\
\hline 1 & 28 & $(140,-10)$ & $(0,70)$ & 61 & ruim \\
\hline 2 & 58 & $(140,-30)$ & $(80,90)$ & 75 & ruim \\
\hline 3 & 42 & $(100,-50)$ & $(10,90)$ & 84 & regular \\
\hline 4 & 50 & $(140,0)$ & $(80,90)$ & 82 & regular \\
\hline 5 & 29 & $(130,-30)$ & $(10,80)$ & 68 & ruim \\
\hline 6 & 30 & $(100,-30)$ & $(80,90)$ & 92 & bom \\
\hline 7 & 12 & $(110,-40)$ & $(0,30)$ & 59 & ruim \\
\hline 8 & 12 & $(110,-80)$ & $(80,90)$ & 50 & ruim \\
\hline 9 & 12 & $(130,-10)$ & $(80,90)$ & 91 & bom \\
\hline 10 & 12 & $(120,-15)$ & $(80,90)$ & 92 & bom \\
\hline 11 & 48 & $(140,0)$ & $(80,90)$ & 76 & ruim \\
\hline 12 & 69 & $(140,0)$ & $(80,80)$ & 96 & excelente \\
\hline 13 & 54 & $(140,-20)$ & $(80,80)$ & 96 & excelente \\
\hline 14 & 12 & $(140,0)$ & $(70,90)$ & 84 & regular \\
\hline 15 & 13 & $(140,-10)$ & $(80,90)$ & 73 & ruim \\
\hline 16 & 68 & $(140,0)$ & $(80,90)$ & 93 & excelente \\
\hline 17 & 23 & $(100,-45)$ & $(80,50)$ & 77 & ruim \\
\hline 18 & 44 & $(140,-20)$ & $(10,50)$ & 77 & ruim \\
\hline 19 & 51 & $(130,-10)$ & $(80,90)$ & 92 & bom \\
\hline 20 & 29 & $(100,-30)$ & $(80,90)$ & 96 & excelente \\
\hline 21 & 84 & $(130,-15)$ & $(10,80)$ & 86 & regular \\
\hline 22 & 51 & $(130,-25)$ & $(80,90)$ & 89 & regular \\
\hline 23 & 32 & $(140,-30)$ & $(80,90)$ & 64 & ruim \\
\hline 24 & 42 & $(120,-20)$ & $(50,70)$ & 81 & regular \\
\hline 25 & 24 & $(140,0)$ & $(80,90)$ & 97 & excelente \\
\hline 26 & 26 & $(140,-10)$ & $(80,90)$ & 96 & excelente \\
\hline 27 & 16 & $(140,0)$ & $(80,90)$ & 100 & excelente \\
\hline 28 & 12 & $(130,-30)$ & $(60,70)$ & 74 & ruim \\
\hline 29 & 12 & $(110,-30)$ & $(80,60)$ & 64 & ruim \\
\hline 30 & 6 & $(140,-10)$ & $(90,80)$ & 89 & regular \\
\hline
\end{tabular}


TABELA 4

Tipos de lesões e resultados funcionais obtidos segundo critérios de Bruce et al

\begin{tabular}{|c|c|c|c|c|c|c|}
\hline & \multicolumn{2}{|c|}{ Satisfatórios } & \multicolumn{3}{|c|}{ Insatisfatórios } & \multirow[t]{2}{*}{ Total } \\
\hline & Excelente & Bom & Regular & & uim & \\
\hline \multicolumn{7}{|c|}{ Fratura da ulna } \\
\hline Tipo I & - & - & - & & $(100 \%)$ & $1(100 \%)$ \\
\hline Tipo II & $3(21 \%)$ & $2(15 \%)$ & $3(21 \%)$ & 6 & $(43 \%)$ & $14(100 \%)$ \\
\hline Tipo III & $4(27 \%)$ & $2(13 \%)$ & $4(27 \%)$ & 5 & $(33 \%)$ & $15(100 \%)$ \\
\hline Total & $7(23 \%)$ & $4(14 \%)$ & $7(23 \%)$ & 12 & $(40 \%)$ & $30(100 \%)$ \\
\hline \multicolumn{7}{|c|}{ Luxação da cabeça do rádio } \\
\hline Anterior & $1(9 \%)$ & $1(9 \%)$ & $4(36 \%)$ & 5 & $(46 \%)$ & $11(100 \%)$ \\
\hline Posterior & $6(31 \%)$ & $3(16 \%)$ & $3(16 \%)$ & 7 & $(37 \%)$ & $19(100 \%)$ \\
\hline Total & $7(23 \%)$ & $4(14 \%)$ & $7(23 \%)$ & 12 & $(40 \%)$ & $30(100 \%)$ \\
\hline \multicolumn{7}{|c|}{ Fratura da cabeça do rádio } \\
\hline Ro & $3(21 \%)$ & $3(21 \%)$ & $4(29 \%)$ & 4 & $(29 \%)$ & $14(100 \%)$ \\
\hline R1 & $1(50 \%)$ & $1(50 \%)$ & - & & - & $2(100 \%)$ \\
\hline $\mathrm{R} 2$ & - & - & $1(20 \%)$ & 4 & $(80 \%)$ & $5(100 \%)$ \\
\hline R3 & $3(33 \%)$ & - & $2(22 \%)$ & 4 & $(45 \%)$ & $9(100 \%)$ \\
\hline Total & $7(23 \%)$ & $4(14 \%)$ & $7(23 \%)$ & 12 & $(40 \%)$ & $30(100 \%)$ \\
\hline \multicolumn{7}{|c|}{ Fratura do coronóide } \\
\hline $\mathrm{CO}$ & $4(29 \%)$ & $1 \quad(7 \%)$ & $3(21 \%)$ & 6 & $(43 \%)$ & $14(100 \%)$ \\
\hline $\mathrm{C} 1$ & $1(33 \%)$ & $1(33 \%)$ & $1(33 \%)$ & & - & $3(100 \%)$ \\
\hline $\mathrm{C} 2$ & $1(50 \%)$ & $1(50 \%)$ & - & & - & $2(100 \%)$ \\
\hline $\mathrm{C} 3$ & $1(12 \%)$ & $1(12 \%)$ & $2(25 \%)$ & 4 & $(50 \%)$ & $8(100 \%)$ \\
\hline $\mathrm{C} 4$ & - & - & $1(33 \%)$ & 2 & $(67 \%)$ & $3(100 \%)$ \\
\hline Total & $7(23 \%)$ & $4(14 \%)$ & $7(23 \%)$ & 12 & $(40 \%)$ & $30(100 \%)$ \\
\hline
\end{tabular}

A relação entre o tipo de fratura segundo Chick et $a l^{(1)}$ e os resultados pode ser vista na tabela 4.

Oito pacientes $(27 \%)$ evoluíram com complicações: cinco casos de pseudartrose da ulna (16\%), um caso $(3 \%)$ de sinostose radioulnar proximal e dois casos $(7 \%)$ de diminuição da amplitude de movimento (ADM) considerada funcional ${ }^{(7)}$. Dos cinco casos de pseudartrose, três deles tiveram fraturas expostas e um tinha uma fratura da ulna fechada, cominutiva.

Os dois casos que evoluíram com diminuição da ADM funcional foram submetidos à liberação articular do cotovelo e obtiveram amplitude de flexão-extensão considerada, por Morrey et al, como funcional( ${ }^{(7)}$.

O caso 21 desenvolveu sinostose radioulnar proximal e, após duas cirurgias para ressecção da sinostose e liberação do cotovelo, obteve amplitude de flexão-extensão funcional, embora a amplitude de pronossupinação seja menor que aquela considerada funcional ${ }^{(7)}$.

Dos 30 casos analisados no trabalho, 15 foram submetidos a mais de um procedimento cirúrgico: em três casos de fraturas expostas a fixação externa inicial foi posteriormente convertida para síntese interna. Em cinco casos foi necessária a correção de pseudartrose da ulna. Em dois casos de importante diminuição da ADM funcional e em um caso de sinostose radioulnar proximal, foi indicada a liberação articular. Nos quatro casos restantes, foi necessária a remoção do material de síntese devido à proeminência e/ou desconforto relatado pelos pacientes.

Com relação à avaliação estatística, estudamos as seguintes variáveis:

\section{Exposição da fratura e resultados}

Quando comparamos os resultados com a exposição da fratura, obtemos uma diferença estatisticamente significativa ( $\mathrm{p}$ $=0,010)$, ou seja, os resultados estão relacionados com a exposição do foco de fratura (tabela 5).

\section{Sexo e resultados}

Comparando sexo com os resultados, obtivemos $\mathrm{p}=0,074$. Embora não houvesse diferença estatisticamente significativa, encontramos tendência aos melhores resultados nos pacientes do sexo feminino e piores resultados em pacientes masculinos (tabela 5). 


\section{TABELA 5}

Resultados da análise estatística pelo teste exato de Fisher

\begin{tabular}{lll}
\hline Exposição da fratura & X Resultados & $\mathrm{p}=0,010$ \\
Sexo & X Resultados & $\mathrm{p}=0,074$ \\
Mecanismo de trauma & X Resultados & $\mathrm{p}=0,035$ \\
Faixa etária & X Mecanismo de trauma & $\mathrm{p}=0,052$ \\
Sexo & X Mecanismo de trauma & $\mathrm{p}=0,035$ \\
\hline
\end{tabular}

\section{Mecanismo de trauma e resultados}

Em relação ao mecanismo de trauma e resultados, a análise estatística foi de $p=0,053$, ou seja, não houve diferença estatisticamente significativa, mas observamos tendência a piores resultados com trauma de alta energia e melhores resultados nos casos decorrentes de trauma de baixa energia (tabela 5).

\section{Faixa etária e mecanismo de trauma}

Ao compararmos a faixa etária e mecanismo de trauma, tivemos $p=0,052$. Não houve diferença estatisticamente significativa, porém houve tendência à associação entre pacientes idosos e trauma de baixa energia (tabela 5).

\section{Sexo e mecanismo de trauma}

Quando comparamos sexo e mecanismo de trauma, não houve diferença estatisticamente significativa $(p=0,053)$. Contudo, houve tendência de as pacientes do sexo feminino sofrerem trauma de baixa energia (tabela 5).

\section{DISCUSSÃO}

Neste estudo, foram avaliadas apenas lesões complexas da região proximal do antebraço. Essas lesões devem, de fato, ser consideradas à parte, dados sua raridade, gravidade e alto índice de complicações ${ }^{(2,8-11)}$. Teasdall et al relataram que o tratamento das fraturas do cotovelo apresenta maior dificuldade que qualquer outra fratura periarticular, devido às múltiplas articulações envolvidas na mobilidade normal do cotove$10^{(9)}$.

Em nossa casuística, não houve predominância das FLEPA em relação ao sexo e ao lado dominante. Alguns autores, entretanto, observaram maior incidência no sexo masculino ${ }^{(3,9,11)}$. Egol et al, ao contrário, relataram predominância do sexo feminino $^{(12)}$. Quanto à idade dos pacientes, assim como na maioria das séries ${ }^{(1,9-12)}$, nesta observamos ampla distribuição por faixa etária. Chick et al ${ }^{(2)}$ salientaram que esse tipo de fratura acomete jovens e idosos em proporção semelhante. Quanto ao mecanismo do trauma, $43 \%$ dos casos sofreram traumatismos de alta energia e os $57 \%$ restantes, de baixa energia.
Embora a análise estatística não tenha estabelecido relação definitiva entre sexo dos pacientes, mecanismo de trauma e qualidade dos resultados, houve tendência de associação entre sexo feminino, traumatismo de baixa energia e bons resultados, bem como entre sexo masculino, traumatismo de alta energia e resultados ruins. Neste estudo, $27 \%$ dos pacientes tiveram fraturas expostas e sua ocorrência teve relação estatisticamente significativa com mau resultado, assim como relatado por Chick et $a l^{(2)}$. Ao contrário, Givon et al não observaram tal relação ${ }^{(8)}$.

O tratamento padrão para as FLEPA consiste em redução aberta e fixação interna estável da fratura, que permita mobilização precoce e rápida recuperação funcional ${ }^{(3,9-10,13-14)}$. Em nossos pacientes, a fratura foi reduzida por via posterior, que permite acesso global ao cotovelo, e fixada com placa de compressão dinâmica (DCP) aplicada à crista da ulna, exceto em um caso (caso 30), cuja fratura epifisária do olécrano foi fixada com banda de tensão. Embora também possa ser aplicada sobre as faces lateral ou medial da ulna, os melhores resultados biomecânicos são obtidos com a colocação da placa sobre a crista da ulna, visto que as forças de tensão posteriores concentram-se no ápice da região proximal da ulna ${ }^{(10)}$.

Ainda há controvérsias no que tange à classificação das FLEPA. Desde que Bado ${ }^{(15)}$ agrupou as lesões de Monteggia em quatro tipos principais, muitas outras classificações têm sido propostas ${ }^{(3,16-18)}$. Chick et al, durante o estudo de 38 pacientes com fraturas complexas da região proximal do antebraço desenvolveram uma classificação baseada nas características anatômicas dessas lesões e correlacionaram com o prognóstico ${ }^{(1)}$. Observaram que são fatores de mau prognóstico: a) fraturas da ulna que acometem a fossa sigmóide (tipos I e II); b) desvio posterior da extremidade proximal do rádio; c) fratura da apófise coronóide, especialmente as cominutivas; d) fratura da extremidade proximal do rádio, especialmente as cominutivas ${ }^{(1)}$. Essa parece ser, a nosso ver, a classificação mais completa, por permitir a combinação entre diferentes itens. Entretanto, não encontramos relação estatisticamente significativa entre os parâmetros da classificação e o prognóstico dos pacientes que estudamos.

Chick et al observaram maus resultados em relação com os fatores prognósticos apontados como negativos ${ }^{(2)}$. Autores concordam que a presença de fratura da cabeça de rádio piora o prognóstico dos pacientes com fratura da região proximal do antebraço $^{(1,8,10,14,19)}$. Quanto à direção do desvio da luxação do rádio, Givon et al observaram pior prognóstico nos pacientes com desvio anterior ${ }^{(8)}$, embora Egol et al e Jupiter et al tenham relatado pior prognóstico naqueles com desvio poste- 
rior $^{(12,19)}$. Em nosso trabalho, observamos a predominância do desvio posterior da extremidade proximal do rádio e sua evolução desfavorável ${ }^{(10,14)}$. Ring et al também observaram relação entre a presença de fratura do processo coronóide e mau prognóstico e salientaram a importância de obter redução anatômica dessa fratura, apesar da dificuldade de realizá-la ${ }^{(10)}$.

Em um dos casos que estudamos, encontramos associação entre luxação ulnoumeral posterior, fratura metaepifisária da ulna, fratura multifragmentar da cabeça radial e fratura da base do processo coronóide. Trata-se de uma variante complexa e incomum da lesão de Monteggia posterior, descrita inicialmente por Preston et al, de tratamento difícil e prognóstico reservado ${ }^{(20)}$.

Em nosso estudo identificamos nove pacientes (29\%) que evoluíram com complicações: cinco casos de pseudartrose da

\section{REFERÊNCIAS}

1. Chick G, Court C, Nordin JY. Fractures complexes de l'extrémité supérieure des deux os de l'avant-bras chez l'adulte: Proposition d'une nouvelle classification. Rev Chir Orthop Reparatrice Appar Mot. 2001; 87(8):765-72.

2. Chick G, Court C, Nordin JY. Fractures complexes de l'extrémité supérieure des deux os de l'avant-bras chez l'adulte: Étude rétrospective de 38 cas. Rev Chir Orthop Reparatrice Appar Mot. 2001;87(8):773-85.

3. Müller ME. A classificação compreensiva das fraturas dos ossos longos. In: Müller ME, Schneider R, Allgower M, Willenegger H. Manual de osteossíntese. São Paulo: Manole; 1990. p. 118-50.

4. Perron AD, Hersh RE, Brady WJ, Keats TE. Orthopedic pitfalls in the ED: Galeazzi and Monteggia fracture-dislocation. Am J Emerg Med. 2001; 19(3):225-8

5. Mohan K. Myositis ossificans traumatica of the elbow. Int Surg. 1972; 57(6):475-8

6. Bruce HE, Harvey JP, Wilson JC. Monteggia fractures. J Bone Joint Surg Am. 1974;56(8):1563-76.

7. Morrey BF, Askew LJ, Chao EY. A biomechanical study of normal functional elbow motion. J Bone Joint Surg Am. 1981;63(6):872-7.

8. Givon U, Pritsch M, Levy O, Yosepovich A, Amit Y, Horoszowski H. Monteggia and equivalent lesions. A study of 41 cases. Clin Orthop Relat Res. 1997;(337):208-15.

9. Teasdall R, Savoie FH, Hughes JL. Comminuted fractures of the proximal radius and ulna. Clin Orthop Relat Res. 1993;(292):37-47.

10. Ring D, Jupiter JB, Simpson NS. Monteggia fractures in adults. J Bone Joint Surg Am. 1998;80(12):1733-44.

11. Eglseder WA, Zadnik M. Monteggia fractures and variants: review of distribution and nine irreducible radial head dislocations. South Med J. 2006;99(7):723-7. ulna (16\%), um caso (3\%) de sinostose radioulnar proximal e dois casos $(10 \%)$ de rigidez. Todas essas complicações são amplamente relatadas na literatura científica ${ }^{(2,9-10,13,21)}$ e refletem a gravidade e dificuldade no tratamento das fraturas complexas da região proximal do antebraço.

\section{CONCLUSÃO}

Os tratamentos das FLEPA têm, em sua maioria, resultados insatisfatórios, principalmente nas fraturas expostas, fato estatisticamente significativo em nosso estudo.

Não foi possível definir outros fatores prognósticos. No entanto, encontramos tendência aos melhores resultados nos pacientes do sexo feminino, nos idosos e nas vítimas de traumas de baixa energia.

12. Egol KA, Tejwani NC, Bazzi J, Susarla A, Koval KJ. Does a Monteggia variant lesion result in a poor functional outcome? A retrospective study. Clin Orthop Relat Res. 2005;(438):233-8.

13. Bailey CS, MacDermid J, Patterson SD, King GJ. Outcome of plate fixation of olecranon fractures. J Orthop Trauma. 2001;15(8):542-8.

14. Simpson NS, Goodman LA, Jupiter JB. Contoured LCDC plating of the proximal ulna. Injury. 1996;27(6):411-7.

15. Bado JL. The Monteggia lesion. Clin Orthop Relat Res. 1967;50:71-86.

16. Trillat A, Marsan C, Lapeyre B. Classification et traitement des fractures de Monteggia. A propos de 36 observations. Rev Chir Orthop Reparatrice Appar Mot. 1969;55(7):639-57.

17. Beaufils P, Audren JL, Lortat-Jacob A, Benoit J, Perreau M, Ramadier JO. Traumatismes complexes de l'extrémité supérieure des deux os de l'avant-bras. Rev Chir Orthop Reparatrice Appar Mot. 1983;69(4):30316.

18. Heim U. Les fractures associées du radius et du cubitus au niveau du coude chez l'adulte. Analyse de 120 dossiers ayant un recul d'un an et plus. Rev Chir Orthop Reparatrice Appar Mot. 1998;84(2):142-53.

19. Jupiter JB, Leibovic SJ, Ribbans W, Wilk RM. The posterior Monteggia lesion. J Orthop Trauma. 1991;5(4):395-402.

20. Preston CF, Chen AL, Wolinsky PR, Tejwani NC. Posterior dislocation of the elbow with concomitant fracture of the proximal ulnar diaphysis and radial head: a complex variant of the posterior Monteggia lesion. J Orthop Trauma. 2003;17(7):530-3.

21. Strauss EJ, Tejwani NC, Preston CF, Egol KA. The posterior Monteggia lesion with associated ulnohumeral instability. J Bone Joint Surg Br. 2006;88(1):84-9. 